\title{
X-SHOOTER SLIT OBSERVATIONS OF GRB HOST GALAXIES
}

\author{
S.D. Vergani ${ }^{1,2}$
}

\begin{abstract}
The Italian-French X-shooter GRB host program started at the end of 2009 and allowed us to collect the spectra of about 30 host galaxies in the 300-2400 $\mathrm{nm}$ range from a redshift of about $\mathrm{z}=0.1$ to $\mathrm{z}=2.7$. We are using these spectra to retrieve information on the host metallicities, star formation rates and extinctions. An accurate slit positioning allowed us to discover in some cases close-by galaxies at the same redshift than the GRB host. Thanks to the comparison of the afterglow and host spectra, it is possible to assess for possible interactions of these systems. We also looked for the counterparts of the foreground absorbers present in the afterglow spectra, but we obtained very few identifications.
\end{abstract}

\section{Introduction}

The X-shooter spectrograph is the first of the ESO/VLT second generation instruments (D'Odorico et al. 2004; Vernet et al. 2011) and has the unique capability to produce spectra covering simultaneously a spectral range from $\sim 3000$ to $24000 \AA$. The consortium that built the instrument had access to GTO (Guaranteed Observation Time) extended over 3 years (2009-2012). Within the GTO we developed our Italian-French program dedicated to the study of the host galaxies of long gamma-ray bursts (LGRB). Thanks to the unique spectral range coverage and sensitivity of the X-shooter spectrograph we can now extend LGRB host studies to high redshift (previously mainly limited to $z<1$ ) and determine the properties (star formation rate, metallicity, extinction...) of a larger sample of these objects. Our program is divided into two sub-programs: one dedicated to slit observations of LGRB hosts at $z>0.8$ and the other devoted to IFU (Integral Field Unit) observations of $10 \mathrm{GRB}$ hosts at $z<0.5$. This is the first IFU survey of LGRB hosts.

The following sections are dedicated to the slit program only.

${ }^{1}$ INAF, Osservatorio Astronomico di Brera, via E. Bianchi 46, 23807 Merate, Italy

2 GEPI, Observatoire de Paris, CNRS, Univ. Paris Diderot, 5 place Jules Janssen, 92195 Meudon, France 


\section{The slit spectra of the Italian-French X-shooter LGRB host program}

To date we collected 24 slit spectra: 9 for LGRB hosts at $0.8<z<1.5$ and 15 at $z>1.5$. The data reduction of the sample is completed and the data analysis is ongoing. Some highlights of this ongoing study will be presented in the following sections. Emission lines of the hosts are detected in 15/24 cases (see Fig. 1). Considering the sensitivity of X-shooter and supposing that the hosts are star forming, the non-detections imply that also at high redshift many LGRB hosts are faint dwarf galaxies. Nonetheless the detected lines can be also very strong, indicating that the host galaxy population covers a variety of types of star forming galaxies.

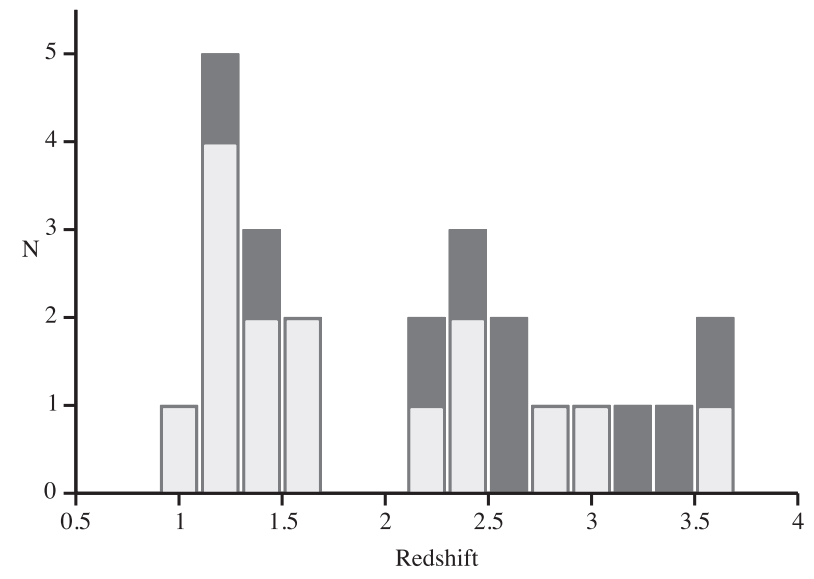

Fig. 1. Redshift distribution of the host galaxies observed in our GTO program (black). The superposed white bars represent the galaxies for which emission lines have been detected.

\section{Mass-metallicity relation}

The study of GRB hosts can bring useful information to galaxy evolution studies (see Savaglio 2012a and Savaglio 2013, these proceedings). LGRB hosts form a sample of galaxies not selected by luminosity, that can be complementary to those of current surveys of galaxies. It has been shown that at $z<1$ their massmetallicity relation follows a trend with a significant offset from the relation found from the surveys of emission line galaxies (Han et al. 2010; Levesque et al. 2010). In order to explain this behavior and to build a complete picture of galaxy evolution, it is important to increase the LGRB host sample to confirm this result and to determine if and how it evolves at higher redshift. The preliminary mass and metallicity values for three hosts of our sample at $z>1.5$ are in agreement with the mass metallicity relation found by Erb et al. (2006) for star forming galaxies at $z \sim 2$ (Fig. 2). With the whole set of data we should be able to assess if the agreement is confirmed. 


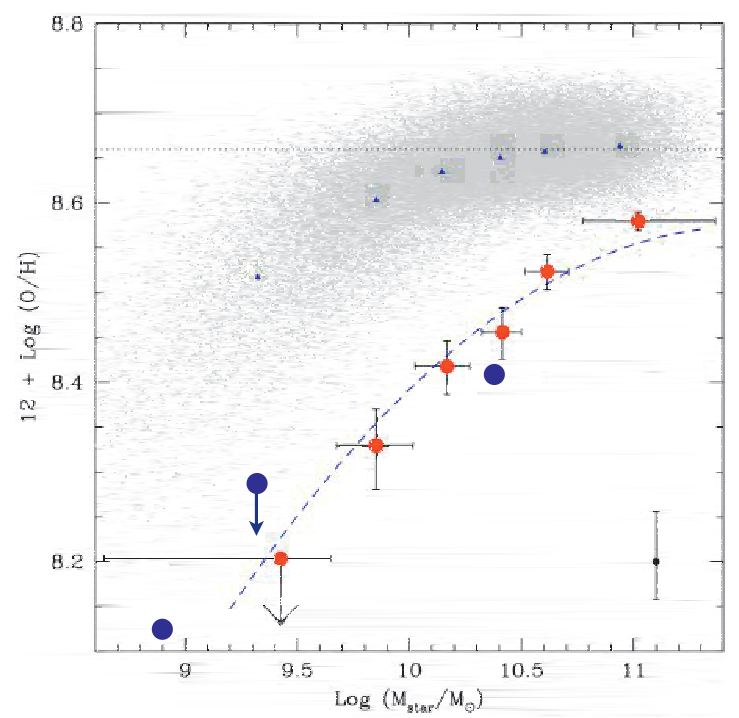

Fig. 2. Mass-metallicity plot for SDSS field star forming galaxies (grey small dots, upper part of the plot) and star forming galaxies at $z \sim 2$ (red dots). The error bar in the lower right corner shows the calibration uncertainty. From Erb et al. (2006). Blue dots represent the preliminary values for three LGRB hosts at $z>1.5$ of our X-shooter sample.

\section{The host galaxies of dark GRBs}

To date the sample of LGRB hosts is biased against dark GRBs. Within our program we are observing also some dark GRB host galaxies, such as the one of GRB 070306 at $z \simeq 1.5$. The afterglow of this GRB showed an extinction corresponding to $\mathrm{A}_{\mathrm{V}}=5.5 \pm 0.5$ (Jaunsen et al. 2008). The X-shooter spectrum of the host shows many emission lines, from the [OII] doublet to the $[\mathrm{SII}]$ one (see Fig. 3). Our preliminary results indicate that the host galaxy has a star formation rate $\mathrm{SFR} \simeq 100 \mathrm{M}_{\text {sun }} \mathrm{yr}^{-1}$, a metallicity of $12+\log (O / H) \sim 8.4$ and an $\mathrm{A}_{\mathrm{V}}=1.3 \pm 0.2$ (considering a SMC extinction curve; similar values are obtained using MW or LMC curves), much less that the amount found for the afterglow extinction, and more than the value retrieved from the host spectral energy distribution (Krühler et al. 2011). These results, added to those reported by other groups (see also Krühler et al. 2011), suggest that dark GRB hosts extend the host population towards more massive galaxies with higher SFR.

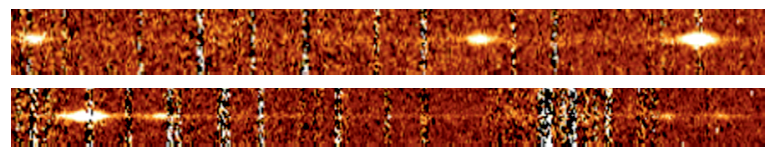

Fig. 3. 2D spectrum (NIR X-shooter arm) of the host of GRB 070306. The emission lines of $\mathrm{H} \beta,[\mathrm{OIII}] 5007$ doublet (top panel), $\mathrm{H} \alpha,[\mathrm{NII}]$ and [SII] doublet (bottom panel) are shown. 


\section{Looking for foreground absorber counterparts}

The surveys of strong MgII intervening absorbers along GRB lines of sight gave the surprising result of an excess of these systems compared to QSO lines of sight (e.g.: Prochter et al. 2006; Vergani et al. 2009). To study the origin of the gas traced by the MgII absorbers, it is of great interest to enlarge the sample of the galaxies responsible for the MgII absorptions towards GRBs and compare their properties with the sample detected for QSO absorbers. Hence, when possible, we placed the slit in order to cover other galaxies present in the GRB field, close-by to the host galaxy. We identified two counterparts only. The strong MgII absorbers are thought to be associated with star forming galaxies (e.g.: Zibetti et al. 2007) and their impact parameters should be smaller for stronger systems (see Schulze et al. 2012 and references therein). The non-detection of the counterparts in our search can be at odds with these assumptions, but a deeper analysis is needed.

\section{Joining emission and absorption studies: Hosts interaction with other galaxies}

Some of the galaxies targeted in our foreground absorber counterpart search turned out to be galaxies at the same redshift of the GRB (e.g.: Fig. 4). For $40 \%$ of the $z>1.3$ galaxies in our sample, we found evidences for interactions with another galaxy in the field, at distances up to $\sim 40 \mathrm{kpc}$ (see also Savaglio et al. 2012b). The combination of the study of the absorption features present in the afterglow spectra and the emission lines of the host galaxy spectra, makes possible to build a more complete picture of the properties of the host and potentially also to retrieve some spatial information on the host gas distribution and on the interaction (see Chen 2012). We can use our data to carry out this kind of studies. An example is the ongoing work on the field of the GRB 021004 (see Vergani et al. 2011).

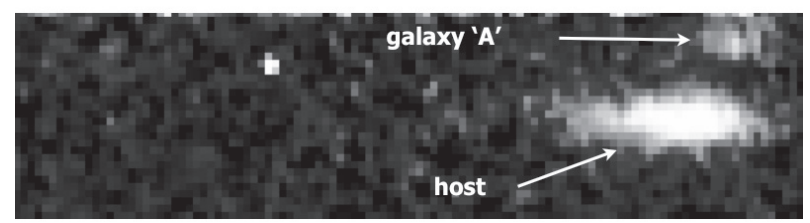

Fig. 4. Section of the NIR 2D spectrum of the GRB 021004 host, showing the [OIII] $\lambda 5007 \AA$ lines of the GRB host and the close-by galaxy A at $\sim 15 \mathrm{kpc}$. This detection together with the profile shape and spatial extension of the host Ly $\alpha$ emission line (see Vergani et al. 2011) suggests a possible interaction between these two galaxies. The high velocity blue-shifted absorption features present in the afterglow spectra could be then due to some outflowing gas connected with this interaction. 
On behalf of the Italian-French X-shooter GRB host collaboration. Based on observations made with ESO Telescopes at Paranal Observatory under programmes ID 084.A-0631, 085.A-0795, 086.A-0874, 087.A.0451 and 089.A-0843 (PIs: S. Piranomonte and H. Flores).

\section{References}

Chen, H.-W., 2012, MNRAS, 419, 3039

D'Odorico, S., Andersen, M.I., Conconi, P., et al., 2004, Soc. Photo-Opt. Instrum. Eng. (SPIE) Conf. Ser., ed. A.F.M. Moorwood \& M. Iye, Vol. 5492, 220

Erb, D.K., Shapley, A.E., Pettini, M., et al., 2006, ApJ, 644, 813

Han, X.H., Hammer, F., Liang, Y.C., et al., 2010, A\&A, 514, A24

Jaunsen, A.O., Rol, E., Watson, D.J., et al., 2008, ApJ, 681, 453

Krühler, T., Greiner, J., Schady, P., et al., 2011, A\&A, 534, A108

Levesque, E.M., Kewley, L.J., Berger, E., \& Jabran Zahid, H., 2010, AJ, 140, 1557

Prochter, G.E., Prochaska, J.X., Chen, H.-W., et al., 2006, ApJ, 648, L93

Savaglio, S., 2012, Astron. Nachr., 333, 480

Savaglio, S., Rau, A., Greiner, J., et al., 2012, MNRAS, 420, 627

Schulze, S., Fynbo, J.P.U., Milvang-Jensen, B., et al., 2012, A\&A, 546, A20

Vergani, S.D., Petitjean, P., Ledoux, C., et al., 2009, A\&A, 503, 771

Vergani, S.D., Piranomonte, S., Petitjean, P., et al. 2011, Astron. Nachr., 332, 292

Vernet, J., Dekker, H., D’Odorico, S., et al., 2011, A\&A, 536, A105

Zibetti, S., Ménard, B., Nestor, D.B., et al., 2007, ApJ, 658, 161 
